\title{
„Die Sprache hat also ihren Ort."Zur Mehrsprachigkeit von Maja Haderlaps Roman Engel des Vergessens
}

\author{
DIETER NEIDLINGER \\ SILKE PASEWALCK
}

\begin{abstract}
Die Sprache hat also ihren Ort." On Multilingualism in Maja Haderlap's Novel Engel des Vergessens. Based on philological research on multilingualism and with regard to Maja Haderlap's literary work in general this article deals with the specific form of multilingualism that can be observed in her novel Engel des Vergessens (2011). Maja Haderlap, born 1961 in Bad Eisenkappel/Železna Kapla, Carinthia (region in southern Austria), grew up with two languages, Slovenian and German. The authors of the article pursue the question to what degree her literary work and especially her novel can be characterised as multilingual and what kind of poetic multilingualism can be found there. They focus on the novel's narrative and on the use of language(s), with a short historical excursus on the Slovenian minority in Carinthia as well as the difficult memory politics in Austria. Maja Haderlap not only writes about the territorial and historical preconditions of multilingualism in Carinthia but also inscribes these conditions in the text itself, characterising both the narrative and the language. Although the novel is the result of a shift from Slovenian to German, its multilingualism can be analysed on different levels: on the level of the relationship between discours and histoire - to refer to Genette's narratological terms -, on the level of cultural codes of the Slovenian language within the novel's German text, and in general with regard to the fact, that the text is written with the modes of expression of Slovenian and German or with the help of the 'no man's land' between the languages. One can therefore - with respect to the terms of philological research - find both obvious and latent multilingualism and, thirdly, one can observe Mehr-Sprachlichkeit, a term that has been defined by Silke Pasewalck in previous articles.
\end{abstract}

Keywords: multilingualism; territorial and historical preconditions of multilingualism; Slovenes in Carinthia; language change; language policy; politics and poetics of memory; Maja Haderlap 
Die Schriftstellerin Maja Haderlap, geboren 1961 in Bad Eisenkappel/Železna Kapla, Österreich, ist mehrsprachig aufgewachsen; in Kindesjahren zunächst in der slowenischen Sprachgemeinschaft im österreichischen Kärnten, wo man in einigen Dorfgemeinschaften noch einsprachig slowenisch geprägt werden konnte. In der Schule eignete sie sich Deutsch als zweite Sprache an, nicht als Fremdsprache, sondern als Sprache der Schule, des Studiums und der österreichischen Öffentlichkeit. In einer Rede bekennt sie: „Die deutsche Sprache ist mir zugewachsen, sie ging mir zur Hand“" (Haderlap 2013: 3). Ihr stehen also zwei Schreibsprachen potentiell zur Verfügung. Doch sind die Texte von Maja Haderlap ebenfalls mehrsprachig? " „Mein literarisches Werk ist zweisprachig“, urteilt die Autorin in ihrer Selbstvorstellung vor der Deutschen Akademie für Sprache und Dichtung (Haderlap 2016). Ist hier mit Mehrsprachigkeit gemeint, dass sie monolinguale Texte in der einen als auch in der anderen Sprache schreibt, oder dass die Sprachen innerhalb ein und desselben Textes wechseln?

Diese Frage soll im Folgenden an Maja Haderlaps Roman Engel des Vergessens (2011) angelegt werden mit dem Ziel, die spezifische Mehrsprachigkeit dieses Textes herauszuarbeiten. Der Romananalyse sei eine genauere Bestimmung und Kontextualisierung von Haderlaps literarischem Schaffen unter dem Blickwinkel der Mehrsprachigkeit vorangestellt. In die Romananalyse ist ein kurzer historischer Exkurs zur slowenischen Minderheit in Kärnten eingelagert.

1 Die Frage nach der Mehrsprachigkeit von Texten beantwortet die Forschung unterschiedlich. Manfred Schmeling möchte Autoren, die zwar „mehrere Sprachen sprechen, aber trotzdem ihre konkreten Texte monolingual gestalten", ausschließen (Schmeling 2004: 225). Die Gegenposition leitet sich aus der radikalen Infragestellung der Einsprachigkeit ab; danach wäre jeder Text mehrsprachig. David Martyns luzide Analyse hat erstens nachgewiesen, dass Mehrsprachigkeit und Einsprachigkeit (und historisch auch das Muttersprachenkonzept) sich jeweils voraussetzen (Martyn 2014: 44) und als solche begriffliche Konstruktionen sind (Martyn 2014: 45). Da es zweitens Texte gibt, die in dieses binäre Raster nicht passen und sich einer ohnehin historisch bedingten Sprachigkeit, also der Zuordnung zu einer oder auch mehreren Sprachen, entziehen, zieht er - im Rückgriff auf Robert Stockhammers Begriff der Sprachigkeit den Schluss, dass es „unterschiedliche Grade an Sprachigkeit [gibt]“ und dass diese „Grade an Sprachigkeit von Wort zu Wort, von Satz zu Satz, von Text zu Text" sowie historisch variieren (Martyn 2014: 49f.). 
„Die Sprache hat also ihren Ort."

\section{Zur Mehrsprachigkeit von Text und Territorium}

Maja Haderlap debütierte 1983 mit Gedichten auf Slowenisch (Žalik pesmi). Es folgten die Gedichtbände Bajalice (1987) und Pesmi Poems (1998). 2011 wechselte sie mit dem Roman Engel des Vergessens die Schreibsprache und verfasste den folgenden Gedichtband langer transit. Gedichte (2014) ebenfalls auf Deutsch.

Im Roman sowie insbesondere in den Gedichten finden sich allerdings Elemente manifester Mehrsprachigkeit. ${ }^{2}$ Im Gedichttitel "!trieste trst triest" (Haderlap 2014: 8) etwa verbinden sich die Namen der ,Stadt der drei Winde' aus dem Italienischen, Slowenischen und Deutschen klanglich zu einem eigenen Idiom und markieren sowohl die Unterschiede als auch die Verwandtschaft der Kulturen, die die Stadt prägten. Hingegen wechselt das Gedicht „barje“ (Haderlap 2014: 32) vom slowenischen Titel ins Deutsche und endet mit dem Wort „Moor", kehrt also zum Titel in Form der deutschen Entsprechung von ,barje zurück. Formen latenter Mehrsprachigkeit bieten ein weites und begrifflich noch nicht sicher abgestecktes Untersuchungsfeld. Grundsätzlich geht es um die vielfältigen Formen der „Mehrsprachigkeit einsprachiger Texte" (Nebrig 2012: 136). ${ }^{3}$ Dies ist bei Haderlap beispielsweise der

2 Bei der manifesten Mehrsprachigkeit kann man zwischen Sprachwechsel und Sprachmischung in einem Text unterscheiden. „Wenn in einem Text Segmente, die unterschiedlichen Idiomen zuzuordnen sind, aufeinanderfolgen", so bezeichnet dies einen Sprachwechsel, hingegen meint Sprachmischung, dass sich Idiome unterschiedlicher Sprachen nicht „einzelnen Segmenten zuordnen“ lassen (Dembeck 2017: 125). Wann genau ein Text das Prädikat,manifest mehrsprachig' verdient, ist nicht abschließend geklärt. Vorgeschlagen wurde etwa, eine signifikante Häufigkeit zum Kriterium zu nehmen. Vgl. Zemanek/Willms 2015: 8 („in signifikanter Menge fremdsprachige [oder auch dialektale] Einsprengsel und Zitate"). Neben der bloßen Quantität wäre ein qualitatives Kriterium denkbar, etwa ob die anderssprachigen Idiome signifikant die Bedeutung verändern oder eine klare ästhetische Funktion haben und nicht nur dekorativ oder kolorierend eingesetzt sind. So stammt etwa bei Haderlaps Gedicht „home“ nur der Titel aus dem Englischen, so dass von einer signifikanten Häufigkeit nicht gesprochen werden kann. Doch entscheidend ist hier, dass das Wort ,home' ein spezifisches Konzept aufruft. Allerdings könnte man sich auch fragen, ob das Wort ,home' nicht ein Beleg für die Problematik der Sprachgrenze(n) ist; kurz gesagt, ob der Idiom-Begriff, wie er in der Mehrsprachigkeitsforschung Verwendung findet, hier überhaupt noch greift.

3 Alexander Nebrig, aus der Warte der Komparatistik und hinsichtlich der Weltliteratur argumentierend, stellt fest: „Mehrsprachigkeit muss nicht immer [...] direkt in den Text eingeschrieben sein, sondern kann auch verdeckt bleiben und doch die Aussageabsicht des Textes lenken." (Nebrig 2012: 136). Weitere terminologische Ausdifferenzierungen stehen bei der latenten Mehrsprachigkeit noch aus. 
Fall, wenn in ihrem Roman offenkundig wird, dass die vielen Gespräche mit der Großmutter, die meistens in indirekter Rede wiedergegeben werden, in der erzählten Welt auf Slowenisch stattfinden. Dies wird nach wenigen Seiten manifest: als das erzählende Mädchen sagt, es habe „sonci gre“ (Haderlap 2011: 9) gerufen, also Slowenisch gesprochen. Oder wenn die Erzählerin als Studentin von ihren Schreibversuchen und ihrem Sprachmangel berichtet und sagt, sie „schreibe auf Slowenisch“ (Haderlap 2011: 175), oder wenn es heißt, „[i]n den Wald zu gehen bedeutet in unserer Sprache nicht nur, Bäume zu fällen, zu jagen oder Pilze zu sammeln“ (Haderlap 2011: 75), so wird damit das Wortkonzept erläutert, das sich aus den spezifischen Kollektiverfahrungen in die Sprache eingeschrieben hat. Weitere Formen der latenten Mehrsprachigkeit sind - um noch zwei weitere Möglichkeiten zu nennen - Metaphern oder Syntaxstrukturen, die kreativ von einer Sprache in eine andere übertragen werden. Bei dem Roman ließe sich etwa überlegen, ob sich der Dual der slowenischen Sprache in die durch Gespräche geprägte Erzählung eingeschrieben hat.

Nimmt man die Produktions- und Rezeptionsbedingungen von Texten hinzu, so eröffnen sich zwei weitere Untersuchungsfelder der Mehrsprachigkeit. In diesem Sinne wäre Haderlaps Werk schon allein deshalb mehrsprachig zu nennen, weil die Autorin aus einer Mehrsprachigkeit heraus schreibt, bzw. weil ihre Werke potentieller Gegenstand der Sprachkontaktforschung sind. Hier wäre zunächst die individuelle Mehrsprachigkeit ${ }^{4} z u$ nennen, wobei kein eindeutiger Fall von bilingualem Erstspracherwerb vorliegt, gleichwohl von einer klaren Bilingualität ausgegangen werden kann. ${ }^{5}$ Obwohl Haderlap bekennt, ihre Zweisprachigkeit selber immer als Bereicherung empfunden zu haben, weist sie zugleich darauf hin, dass ihr die in Kärnten „beheimateten Sprachen als ideologische, politische Kategorie vorgeführt" wurden, ja, als „zwei einander ausschließende Pole, zwischen denen [...] [sie sich] entscheiden müsste" (Haderlap 2011b). Wir haben es demnach in Österreich mit einer spezifischen Situation von territorialer Mehrsprachigkeit ${ }^{6}$ mit ihren eigenen historischen und politischen Implikationen zu tun. Doch auch aktuell ist das Slowenische dort heutzutage trotz

4 Die individuelle bzw. personale Mehrsprachigkeit erscheint auf den ersten Blick für Fragen der Mehrsprachigkeit von literarischen Texten irrelevant. Doch die Texte Haderlaps reflektieren eine Sprachauffassung, die auch Fragen der individuellen Mehrsprachigkeit berührt.

5 Man kann verschiedene Typen der Bilingualität unterscheiden. Vgl. beispielsweise Müller 2006: 44f.

6 Bei der territorialen spricht man auch von der gesellschaftlichen Mehrsprachigkeit neben der individuellen und institutionellen. Zur weiteren Ausdifferenzierung der territorialen Mehrsprachigkeit siehe Claudia Maria Riehl 2004: 53-55. 
„Die Sprache hat also ihren Ort."

verfassungsrechtlich zugesichertem Minderheitenstatus einem Verdrängungsdruck schon einzig durch die Dominanz der deutschen Sprache in Österreich ausgesetzt, der nicht nur Hintergrund ihrer Texte ist, sondern auch Haderlaps Sprachwechsel betrifft. Bei aller Spezifik werden hier selbstredend grundsätzliche Fragen der Sprachkontaktforschung aufgeworfen - so etwa nach den Inklusionsund Exklusionsmechanismen von Sprache, Identitätsfragen, Machtasymmetrien zwischen Sprachen, Ideologisierung und Instrumentalisierung von Sprachen etc. Im Roman findet sich die rhetorische Frage: „Aber ist Friede in dieser Gegend überhaupt heimisch geworden oder tragen die hier gesprochenen Sprachen immer noch Uniform?" (Haderlap 2011: 220). Bei einer derartigen historischen Last und einer solchen Politisierung und den damit einhergehenden Machtasymmetrien und Diskriminierungen kann literarische Mehrsprachigkeit nicht als bloßes ästhetisches Mittel betrachtet werden. ${ }^{7}$

Damit ist das Feld der Mehrsprachigkeit bei Haderlap kursorisch abgesteckt. Die folgende Untersuchung wird sich auf die Frage nach dem Sprachwechsel Haderlaps konzentrieren, wie er sich in Engel des Vergessens eingeschrieben hat, und dabei sämtliche hier genannten Felder berühren. Im Zentrum stehen dabei in erster Linie der Text und die Protagonistin des Romans, nur in zweiter Linie die Autorin selbst. Der Roman erzählt nicht nur das - in die Lebens- und Familiengeschichte eingelagerte - tragische Schicksal der Kärntner Slowenen, sondern auch seine Genese und Intention, die ambivalenten Bedingungen seiner Möglichkeiten, seine eigene Topographie. Haderlap selbst umreißt diese Frage in ihrer Rede Übergänge wie folgt:

Einen nicht auf den ersten Blick erkennbaren Erzählfaden aus meinem Roman „Engel des Vergessens“ möchte ich an dieser Stelle aufnehmen. Es ist die Erzählung vom Verlassen einer Sprache und vom Hinübergleiten in eine andere, die schon immer da war [...], es ist die Geschichte eines Übergangs, einer Metamorphose, eines Verlusts vielleicht [...]. (Haderlap 2013)

\section{Der Roman Engel des Vergessens als Entwicklungsgeschichte}

Der Roman erzählt eine Lebensgeschichte, eine Entwicklungsgeschichte wie ein Kind in dem engen Gebirgstal Lepena aufwächst, als Mädchen die Schule im benachbarten Klagenfurt besucht, zum Studium nach Wien zieht,

\footnotetext{
Eva Schörkhuber weist darauf hin, dass „die politische Dimension und die politische Bedeutung des Buches oftmals gegen seinen poetischen Tonfall, gegen seine Literarizität ausgespielt" werde, und fordert eine Kontextualisierung der Lektüre von Maja Haderlaps Roman Engel des Vergessens ein (Schörkhuber 2017: 114).
} 
im Theater zu arbeiten und Gedichte zu schreiben beginnt. Am Ende deutet sich an, dass die inzwischen erwachsene Frau den Roman, den die Leserinnen und Leser in den Händen halten, schreiben wird. Der Text erzählt also letztlich die Geschichte, wie es dazu kam, dass er geschrieben werden wird. Das bedeutet nicht, dass wir in ihm eine literarische Autobiographie der zum Schreiben gekommenen Autorin sehen, wenngleich hinter der Protagonistin Haderlaps alter ego angenommen werden kann. Die Idee ist vielmehr, dass der Roman seinen (poetologischen) Ausgangspunkt beschreibt.

Die Handlung setzt in der Küche der Großmutter ein, als das Kind noch nicht zur Schule geht, und endet im Jahre 2003, als die Protagonistin nunmehr 41 Jahre alt - das Konzentrationslager Ravensbrück besucht, wo ihre Großmutter, zu der sie eine sehr enge Beziehung hatte, interniert war. Vom Lager, von denen, die „überlebt haben oder in den Lagern ums Leben gekommen sind" (Haderlap 2011: 142), hat die Großmutter ihr erzählt. Erst an ihrem Totenbett wird deren Sohn von seiner traumatischen Erfahrung berichten und sein Schweigen brechen; so erfährt seine Tochter erstmals davon, wie er als zehnjähriger Junge gefoltert wurde und zu den Partisanen in den Wald flüchten musste. Aber zwischen der Großmutter, die über das Geschehene gesprochen hat, dem Vater, der darüber schwieg, und der Mutter, die davon nichts mehr hören wollte, wächst die Tochter auf und muss in einem Land ihren Platz suchen, in dem dieses Leid und Schicksal ihrer Herkunft ein Tabu war und dem offiziellen kulturellen Gedächtnis widersprach. Erst der Roman erschafft diesen Erinnerungsort, indem er die Geschichte erzählt, in der es diesen noch nicht gibt.

Am Anfang ist da nur die Küche, wo die Großmutter dem Kind von ,jenen zwei Jahren ihres Lebens, die sie am tiefsten gezeichnet haben“ (Haderlap 2011: 118) erzählt. Das Kind versteht die Dinge auf seine Art und versteht noch wenig. Die Küche des Hause scheint mit der Küche des KZ in Ravensbrück zu verschmelzen: „Ich höre ihr zu, wenn sie erzählt, für wie viele Menschen sie schon gekocht hat, damals zu Hause, [...] damals, als sie die Kessel gewaschen hat, das war noch ein Glück, sagt sie, dass sie dahin gekommen sei, in die Küche, im Lager" (Haderlap 2011: 6f.). Was ein Partisan sei, fragt sie die Mutter, weil sie weder das Wort kennt noch vom Widerstandskampf der Slowenen etwas weiß: „Hat dir Großmutter wieder Geschichten erzählt?“ (Haderlap 2011: 31), fragt die Mutter, offensichtlich wenig begeistert von dem Einfluss ihrer Schwiegermutter auf das Kind. Selbst streng katholisch, befestigt sie „zwei gerahmte Engelbildchen“ (Haderlap 2011: 13) ${ }^{8}$ über dem Bett ihres

8 Die Figur des Engels fungiert geradezu als Chiffre des Romans. Die beiden katholischen Engelbildchen stehen dem Engel der Geschichte, einer Denkfigur Walter Benjamins, und 
„Die Sprache hat also ihren Ort."

Kindes, um es zu beschützen. Sie möchte die alten Geschichten hinter sich lassen, kauft einen Kühlschrank, der symbolisch für Fortschritt und Wohlstand und Veränderung steht. Zwischen der Großmutter, die den Hof nur für den Gottesdienst oder eine Hochzeit verlässt, und der Mutter, die immer davon redet, fortgehen zu wollen, wächst das Kind auf und wird seinen Weg finden müssen.

\section{Historischer Exkurs ${ }^{9}$}

Nach dem Ersten Weltkrieg entscheidet bei einer Volksabstimmung die Mehrheit - auch der Slowenen - in Kärnten, Teil Österreichs sein zu wollen, und in der Verfassung werden Minderheitenrechte festgeschrieben. Die Slowenen südlich der Grenze werden Teil eines Serbisch-Kroatisch-Slowenischen Staates, aus dem Jugoslawien hervorgehen wird. Nach dem Anschluss Österreichs ans Dritte Reich im März 1938 verschärft sich die Lage der Kärntner Slowenen aufgrund der nationalsozialistischen Sprachenpolitik das Slowenische soll aus dem öffentlichen Leben, so auch den Schulen, verschwinden. Nach dem Angriff der Wehrmacht auf Jugoslawien im April 1941 radikalisieren sich die Maßnahmen zur ,Germanisierung'. Die ersten Deportationen finden statt und es entsteht eine Partisanenbewegung, die grenzübergreifend auch mit Titos Widerstandsbewegung in Jugoslawien kooperiert. Gegen diese geht man mit aller Härte vor, verhängt Sippenhaft, foltert, um Informationen zu erhalten. Wer auch nur in Verdacht gerät, die Partisanen zu unterstützen, wird ermordet oder interniert. ${ }^{10}$ Viele überleben die Lager nicht. Doch nach Kriegsende etabliert sich in Österreich sehr schnell das sogenannte Opfer-Narrativ; demnach war Österreich das ,erste Opfer Hitlers'. Für die Erinnerung an die Kärntner Slowenen, die Widerstand geleistet hatten und von den österreichischen Behörden deportiert, verfolgt, interniert und ermordet worden waren, war in diesem Narrativ kein Platz. Stattdessen

dem titelgebenden Engel des Vergessens gegenüber. Letzterer wird, so heißt es am Ende des Romans, die „Engelsbildchen über [...] [ihrem] Kinderbett endgültig entfern[en].“ (Haderlap 2011: 287)

9 Vgl. zur Geschichte Kärntens auch Burz 2014.

10 So kommt die Großmutter ins KZ Ravensbrück, weil ihr Mann zu den Partisanen ging; ihr Sohn, der spätere Vater der Erzählerin, wird als Zehnjähriger mehrere Tage lang von Polizisten gefoltert. Der Roman referiert fast dokumentarisch die historischen Fakten, so etwa auch das Massaker an Zivilisten auf dem Peršman-Hof wenige Tage vor Kriegsende. Dabei werden Schwarz-Weiß-Schemata wie Heroisierung auf der einen und Schuldzuweisungen auf der anderen Seite tunlichst vermieden und ein differenziertes Bild der Historie entfaltet. 
NEIDLINGER, PASEWALCK

beschwor man eine kommunistische Bedrohung durch Jugoslawien herauf, verdächtigte alle Slowenen in Kärnten der heimlichen Kollaboration und sah schon im Gebrauch der slowenischen Sprache eine Provokation. Den Kampf um die Erinnerung und die Fortsetzung der Verdrängung der slowenischen Sprache bezeichnet man auch recht verharmlosend als Kärntner Sprachenstreit, als dessen Höhepunkt der Streit um die zweisprachigen Ortsschilder 1972 gilt, „wo in Kärnten die zweisprachigen Ortstafeln abgerissen werden“ (Haderlap 2011: 138). Erst mit der Waldheimaffäre 1986 wächst die Kritik an dem Opfermythos und ein Umdenken setzt ein. Waldheim, bis 1992 Präsident in Österreich, war im Krieg an Massakern an der Zivilbevölkerung in Jugoslawien beteiligt. Er hatte diesen Teil seiner Vergangenheit verschwiegen - ganz dem österreichischen Narrativ folgend.

\section{Zum Verhältnis von Sprache und Erzählweise des Romans}

Diesen historischen Hintergrund erschließt sich die Leserin bzw. der Leser, die resp. der die Welt mit dem Blick des Kindes kennenlernt, erst nach und nach. Doch je mehr das Kind erfährt, desto verstörter ist es. Der Besuch mit der Großmutter in einem ehemaligen Gefängnis, wo den Opfern der dort von der Gestapo ermordeten Menschen gedacht wird, geht dem Kind unerträglich nahe:

Der Schrecken hat mittlerweile die Stärke eines Sturms erreicht. Als wir wieder ins Freie treten, habe ich das Gefühl, dass mir die Hälfte des Kopfes fehlt, dass ich von außen betrachtet wie ein Haus aussehe, dem der Sturm das Dach weggerissen hat. (Haderlap 2011: 48)

Obwohl die Erzählung sich immer wieder in solchen poetischen Bildern kristallisiert, in denen die Dinge verdichtet zur Sprache kommen, versteht der Leser die Zusammenhänge noch wenig. Was bleibt, ist das poetische Bild - der Kopf als Haus ohne Dach. Bis auf wenige Prolepsen sind die Entwicklungen des Kindes aus dessen Innensicht dargestellt. Selbst der historisch kundige Leser wird vom Text in die Erfahrung des Kindes zurückversetzt. So wird, als das Mädchen zur Schule kommt und nun Deutsch als weitere Sprache kennenlernt, dies nicht explizit gesagt; vielmehr wird gezeigt, wie die neue Sprache wirkt und dem Kind erscheint:

Ich kann nicht ergründen, was ich wirklich lebe. Meine Gefühle sind nicht mit den Wörtern vertraut, die ich spreche. Konnte ich früher mit den Wörtern 
„Die Sprache hat also ihren Ort."

nach Gegenständen, Gefühlen und Gräsern werfen und sie treffen, prallen die Wörter jetzt von den Gegenständen und Gefühlen ab. (Haderlap 2011: 100)

Doch in der Schule irritieren das Kind nicht nur die neuen Wörter. Es realisiert zudem, dass seine bisherige (slowenische) Welt in der Schule keinen Platz hat.

Den Kindern in den Schulbüchern stößt immer anderes zu. Ich komme darin nicht vor. Ich denke daran, mich aus der Kindheit zurückzuziehen, weil ihr Dach undicht geworden ist, weil ich Gefahr laufe, mit ihr unterzugehen. (Haderlap 2011: 100)

Das weggerissene Dach verbindet hier leitmotivisch Museums- und Schulbesuch. Steht das Museum für die das Kind verstörenden Erzählungen der Großmutter, so steht die Schule für den neuen Sprachraum. Das Kind erfährt eine Grenze, die die Sprache des Romans durch die Chiffre Dach unterläuft. Die semantische Aufladung von Orten der Handlung wie etwa der Küche oder der Kammer der Großmutter, von Landschaften wie dem Wald ${ }^{11}$ oder den engen Gräben im Gebirgstal, die sich geradezu zu einem poetischen Sprachteppich entfalten, erzeugen eine eigene Sprachlandschaft und eigene Bedeutungen, die sich dem Leser nach und nach erschließen. Die Sprache des Romans weiß immer schon mehr als die Erzählung, in der sich die Zusammenhänge erst nach und nach ergeben. So sagt die Sprache gleich zu Anfang, dass die Großmutter die „Bienenkönigin“ und das Kind ihre „Drohne“ sei (Haderlap 2011: 7). Erst hundert Seiten später wird deutlich, wie stark und wodurch die Großmutter das Kind geprägt hat, und die folgende Formulierung wird verständlich:

Großmutters Schlafzimmer ist ein Gedächtnisort, eine Königszelle, [...] eine Brutzelle, in der ich mit Großmutters Nährflüssigkeit gefüttert werde. In dieser Keimzelle werde ich, wie ich erst Jahre später begreifen werde, geformt. (Haderlap 2011: 117)

Die Vorausdeutung, dass die Protagonistin später die Dinge verstehen wird, erklärt, warum die Sprache des Romans, die die Sprache der Autorin sein wird, mehr sagt als das, was aus der Sicht des Kindes erzählt wird. Die Bienen, die der Vater züchtet, sind auch metaphorisch zu verstehen; wenn der Vater hofft, „dass die geschwächten Bienen vom Nachbarvolk aufgenommen werden" (Haderlap 2011: 20), so steht dies gleichsam für die Frage, ob die Slowenen in Österreich eine Heimat finden.

11 Zum Motiv des Waldganges bzw. der Waldgänger, vgl. Pruzzi 2014. 
NEIDLINGER, PASEWALCK

Sobald das Mädchen die Schule besucht, wird ihm vermittelt, als Slowenin in Österreich keinen Platz zu haben. Das Mädchen erfährt, dass eine Grenze gezogen wurde zwischen ihm und den Kindern in den Büchern, zwischen der einen und der anderen Sprache, und es überlegt, ob es „in etwas hinüberwechseln müsste, wofür [...] [es] keinen Begriff habe." (Haderlap 2011: 101)

„Wo ist eigentlich die Grenze“, das fragt das Kind den Vater, nachdem es in die Grundschule gekommen war (Haderlap 2011: 78). Der Vater zeigt ihr, wo die Staatsgrenze verläuft. Diese können sie jedoch nicht nur unbemerkt überqueren; auf der anderen Seite wird auch dieselbe Sprache gesprochen und sie werden herzlich empfangen. Nun erfährt das Kind eine andere Art von Grenze, eine Grenze, die durch das eigene Land verläuft - und bald durch ihr Leben eine Sprachgrenze, bei der die Sprachen ideologisch aufgeladen sind.

Nach dem Willen der Mutter soll das Mädchen auf das Gymnasium geschickt werden, soll also die slowenische Enklave in den Gräben ${ }^{12}$ verlassen. Der Vater ist dagegen und die Uneinigkeit der Eltern überträgt sich auf das Kind. Der Text wechselt plötzlich die Erzählsituation von der Ich-Form zum personalen Erzähler:

Am Abend bleibt das Kind hinter dem Haus auf der Wiese stehen, am geöffneten Tor zur Nacht, die als Königspalast aufgeht über der Landschaft, mit klingendem Sterngefunkel, mit dem Atem des Waldes und dem Plätschern des Baches am Grabengrund. (Haderlap 2011: 133)

Die Paläste stehen den Gräben gegenüber, und das Kind weiß nicht, wohin es gehört. Der Wechsel zur 3. Person kann entwicklungspsychologisch gedeutet werden; die Protagonistin kann zu sich in Distanz treten und über sich selbst reflektieren. Doch im folgenden Abschnitt ${ }^{13}$ deutet sich darin zudem eine Spaltung der Person an, die mehr beinhaltet als eine natürliche psychologische Entwicklung.

Das Mädchen [...] dürfte wohl ich sein, das fremde Ich, das das Weinen entdeckt, als Quelle, die alles aus der Tiefe des Körpers schwemmt, was sich dort angesammelt hat, $[\ldots]$ ein Metall $[\ldots]$, das es vergiftet und nährt. (Haderlap 2011: 134)

12 Gräben oder auch Grape - so nennt man die engen Gebirgstäler in Südkärnten. Dort konzentrierte sich die slowenische Minderheit.

13 Der Roman enthält sehr viele Absätze und ist darüber hinaus noch in 50 Abschnitte von sehr unterschiedlicher Länge untergliedert, die jeweils mit einem Initial beginnen. 
„Die Sprache hat also ihren Ort."

\section{Das Leben im Niemandsland zwischen zwei Welten}

Das Kind wird nicht nur zum Mädchen. Wollte es sich zuvor aus dem „Empfindungskörper zurückziehen“ (Haderlap 2011: 117), aus der Kindheit ausziehen, erkennt es in dieser Nacht, dass ihre Herkunft, die sie ernährt hat, die sie vergiftet hat, ein Teil von ihr ist und bleiben wird. Sie gehört zu den Menschen, die von ihren eigenen Erinnerungen attackiert werden, und sie hat zugleich die Vorstellung, die Vision, zu

etwas Samtigen und Warmen vorzudringen, zu etwas Dunklem und Hellem, das mich zermalmt und versöhnt, das mich das Kind sehen lässt, fern von mir, wie in mir. Von da an bin ich das falsch zusammengewachsene Mädchen, kommt mir vor, das Mädchen mit ausgerenkten Gliedern, mit hochtrabenden Gedanken. (Haderlap 2011: 134)

Sie wird in den anderen Sprach- und Kulturraum einziehen und doch ihre Kindheit mitnehmen und den Traum hegen, die getrennten Welten zu verbinden. Gegen den Willen von Vater und Großmutter wechselt die nun Zehnjährige auf das Gymnasium. Dort entwächst sie den Gräben und entfremdet sich von der Großmutter. Ihre Herkunft, „[d]ie Angst um [den] Vater und die Erzählungen der Großmutter formen sich zu einer von [ihr] sorgsam gehüteten Gedankenwelt" (Haderlap 2011: 143), deren Ort die slowenische Studienbibliothek wird. Dort ist ihr Rückzugsraum, weil das „Slowenische [...] etwas Unerwünschtes im Land“ (Haderlap 2011: 143) ist.

Sie versteckt ihre Herkunft also in sich oder verlagert sie in Rückzugsräume wie die slowenische Bibliothek oder die Literatur. Nach außen hin nimmt sie den verheißungsvollen Weg zum „Königspalast“, zum „Sterngefunkel“ (Haderlap 2011: 133), also den einzigen Weg, der in Österreich Erfolg verspricht, den Weg der Anpassung und Assimilation. Nach der Matura zieht sie nach Wien, studiert Theaterwissenschaft und promoviert. Schließlich arbeitet sie am Theater als Dramaturgin. Sie scheint den Weg der Mutter genommen zu haben und die Erzählungen der Großmutter, die Angstzustände des Vaters und seine posttraumatischen Anfälle hinter sich gelassen zu haben, und am Ziel ihrer „, $[\mathrm{h}]$ eimlichen Gedanken“, die in der Schule als „blank geputzte, zarthafte Wünsche" (Haderlap 2011: 101) in ihrem Kopf zu kreisen begannen, angekommen zu sein. 
NEIDLINGER, PASEWALCK

In Wirklichkeit lebt sie weiterhin in zwei Welten, oder zwischen zwei Welten, zwischen denen sich ein „Niemandsland [erstreckt], in dem man verloren gehen kann“ (Haderlap 2011: 185). ${ }^{14}$

Die Reisen zwischen Wien und meinem Heimatort entwickeln sich zu Zeitexpositionen, zu Fahrten durch unterschiedliche Zeitläufe und Geschichtsvarianten, die nebeneinander existieren. [...] Ich sehe mich zwischen einem dunklen, vergessenen Kellerabteil des Hauses Österreich und seinen hellen, reich ausgestatteten Räumlichkeiten hin- und herpendeln. Niemand in den hellen Räumen scheint zu ahnen [...], dass es in diesem Gebäude Menschen gibt, die von der Politik in den Vergangenheitskeller gesperrt worden sind, wo sie von ihren eigenen Erinnerungen attackiert und vergiftet werden. (Haderlap 2011: 185f.)

Schon in der Schulzeit auf dem Gymnasium war in ihr der Gedanke aufgekommen, über dieses „dunkle[ ], vergessene[ ] Kellerabteil (Haderlap 2011: 185), über das in diesem Land niemand sprechen wollte, zu schreiben. Initialzündung war der Tod der Großmutter. Bei der Totenwache beginnen die Leute zu erzählen, und erstmals kann der Vater seine Geschichte erzählen, und seine Tochter erfährt den Grund für seine Ängste und posttraumatischen Anfälle. Seine Erzählung von Folter und Verfolgung, so hat sie danach „das Gefühl“, sei zu der ihren geworden, als habe er ihr „einen Teil [...] [ihrer] eigenen Geschichte erzählt“ (Haderlap 2011: 155). Leni, die im Hause die Rolle der Großmutter übernommen hatte, als jene im $\mathrm{KZ}$ inhaftiert war, erzählt danach ihre Geschichte, die Angst vor Denunziation und Deportation. Am nächsten Tage hat die Enkelin und Erzählerin beim Blick in den Talgraben erstmals den Gedanken, das Erfahrene, ihre Vergangenheit, ihre Herkunft zur Sprache zu bringen:

Nicht zufällig berührt der Blick die Landschaft. Doch es wird nicht der Wald sein und nicht die Gräben, wo das Schreiben seinen Ausgangspunkt findet. Daher ist es zunächst ein Prozess des Scheiterns. Sie findet keine Sprache: „Meine Sprechversuche bleiben ein kleinlautes Stammeln und Schweigen" (Haderlap 2011: 167). Während des Studiums nimmt sie die Schreibversuche auf Slowenisch wieder auf. Doch die „Sätze sind ungelenk, als

14 Das Wort Niemandsland taucht das erste Mal mit der Schulzeit auf und wird von Haderlap auch in Reden verwendet: In ihrer Bachmannpreisrede heißt es, es sei ein Ort, „den man nur mit äußerster Vorsicht als Niemandsland bezeichnen könnte, weil er selten so unbelebt und unbehaust wirkt, wie der leere, drohende Sicherheitsstreifen zwischen zwei Staaten“ (Haderlap 2013). Der Ausdruck meint also etwas, das ambivalent zu begreifen ist. 
„Die Sprache hat also ihren Ort."

wären sie aus herausgerissenen Buchstabenreihen zusammengestellt." (Haderlap 2011: 175)

\section{Sprachwechsel im Roman}

Maja Haderlap ist es gelungen, den Roman zu schreiben. Sie verschweigt auch nicht, dass sie die Lebensgeschichte mit der Protagonistin teilt, und sie hat für den Roman die Sprache gewechselt, also auf Deutsch - nicht in der Sprache der Täter, vielmehr in der deutschen Literatursprache - ihre Lebensgeschichte, ihre Familiengeschichte, ihre Herkunftsgeschichte zur Sprache gebracht. In einem Gespräch mit Michael Kerbler nennt sie drei Gründe für ihren Wechsel der Schreibsprache. Erstens einen psychologischen Grund: Die deutsche Sprache habe sie geschützt. Deutsch also als Distanzsprache, ${ }^{15}$ die erst ermöglicht, sich dem Stoff zu nähern, weil die Erzählungen der Familie und auch die eigene Erfahrung der Ausgrenzung zu eng mit dem Slowenischen verbunden sind. ${ }^{16}$ Zweitens einen literarischen Grund: Es gibt eine Tradition deutschsprachiger Erinnerungsliteratur, an die sie anschließen konnte. Drittens einen kulturpolitischen Grund: Sie habe in den deutschsprachigen Kulturraum sprechen und etwas bewirken wollen, also in den Raum, der bisher diesen Erinnerungen keinen Platz im kulturellen Gedächtnis zugestanden und der slowenischen Sprache ihr Recht abgesprochen hatte, in ihm beheimatet zu sein.

Doch die Entscheidung, „mutwillig aus einer Schreibsprache auszuziehen“ (Haderlap 2011b), ist höchst ambivalent. In diesem Falle erscheint sie wie ein Verrat an der eigenen Sache. Affirmiert dies nicht den Verdrängungsprozess des Slowenischen durch die deutsche Sprache? Widerspricht es nicht der eigenen Intention, jenen Generationen eine Stimme zu geben, die sich zu ihrer Sprache bekannt und gegen ihre Vernichtung Widerstand geleistet haben? Beide Fragezeichen spricht Haderlap selbst in Reden und Interviews an. Sie weiß nicht nur um die Ambivalenz ihrer Entscheidung, sondern weist zugleich darauf hin, dass man zwar immer nur in einer Sprache schreiben könne, nicht zwischen den Sprachen, dass gleichwohl ihre Texte nach einer Verortung

15 Vgl.: Deutsch hält mich auf Distanz zum Schmerz. Interview mit Maja Haderlap. 2012. Unter https://www.diepresse.com/743059/haderlap-deutsch-halt-mich-auf-distanzzumschmerz (Aufgerufen am 01.08.20).

16 Vgl. hierzu auch ihre Rede Übergänge: „Auch habe ich mich erst über die deutsche Sprache den schmerzlichen Erinnerungen annähern können, habe wieder zum Gedächtnis meines Körpers zurückgefunden und bin zu den Gerüchen der Kindheit zurückgekehrt. Über die Sprache ist mir ein neuer Nervenstrang gewachsen, der die emotionalen Verschüttungen und Verkrustungen überwinden und überlisten konnte." (Haderlap 2013: 4). 
NEIDLINGER, PASEWALCK

suchen, nach einem Ausgangsort, dass sie somit ihre eigene Topographie haben. So spricht sie von der Peripherie des deutschen Sprach- und Kulturraums aus. Sie nimmt die Topographie der Bergkämme, die einen Blick in die Täler und nach Süden bis zum Meer erlauben, zum poetologischen Ausgangspunkt. Und sie schreibt nicht aus einer Sprache, sondern aus ihrer Mehrsprachigkeit heraus.

\section{Zur Mehrsprachigkeit von Text und Sprache}

Dieser Ausgangspunkt des Romans sei abschließend bestimmt. Der erste Satz des Romans spricht von einer schweigenden Gebärde: „Großmutter gibt mir ein Zeichen mit der Hand, ich solle ihr folgen." (Haderlap 2011: 5) Die Geste taucht in der Mitte des Romans erneut auf, wenn es heißt, die „Großmutter [...] [gebe ihr] wiederholt Zeichen, [...] [sie] solle zu ihr kommen, sie müsse [...] [ihr] etwas verraten“ (Haderlap 2011: 117), und ebenso im letzten Satz: „Großmutter gibt mir mit der Hand zu verstehen, dass ich leise sein soll. Nicht so laut, sagt sie, sonst kann man nichts hören." (Haderlap 2011: 287) Hören soll man die Stimmen, die die Großmutter in einem Traum der Enkelin in "gesponnen[en] [...] trichterförmige[n] Baldachinen“ (Haderlap 2011: 287) eingefangen hat. Bezieht man dieses poetische Bild vom Ende auf den ganzen Roman, so wird der Text vom Sprechakt zum potentiellen Hör-Akt und die Erzählerin zur Empfängerin. Da wir sodann in dem Roman die „Stimmen“ (Haderlap 2011: 287) hören, diese ihre Geschichten jedoch auf Slowenisch erzählten, erklingt in dem auf Deutsch geschriebenen Roman die slowenische Sprache. Dies ist weniger paradox, als es zunächst erscheint.

Erstens zeigt sich in dem Roman neben der schon erwähnten manifesten und latenten Mehrsprachigkeit eine weitere Form, wenn man - mit Genette gesprochen - zwischen der Ebene des discours und der der histoire unterscheidet, zweitens sind kulturelle Codes der slowenischen Sprache in den Text übergegangen und drittens ist der Text mehr-sprachlich, da er aus zwei Sprachen bzw. aus dem Niemandsland zwischen den Sprachen heraus geschrieben ist.

Zum ersten Punkt: Im Roman finden Sprachwechsel statt; in der Story gibt es code-switching, Gespräche werden auf Deutsch oder auf Slowenisch geführt und es ist ein Sprachwechsel der Protagonistin in den Roman eingeschrieben. Die meisten Gespräche in der erzählten Welt werden auf Slowenisch geführt, ${ }^{17}$ jedoch, sieht man von slowenischen Einsprengseln ab, auf Deutsch wieder-

17 Die auffälligste Markierung eines Sprachwechsels findet sich im Kontext des Sprachenstreits: „Ich bin ein Mensch, schreit Vater auf Deutsch“ (Haderlap 2011: 138). 
„Die Sprache hat also ihren Ort."

gegeben. Auf der Ebene der histoire ist der Text also über weite Strecken Slowenisch, auf der Ebene des discours ist er Deutsch.

Zum zweiten Punkt: Hier sei auf eine Aussage der Autorin hingewiesen und sodann ein Text-Beispiel gegeben.

Ich habe in Engel des Vergessens den Versuch unternommen, das in der slowenischen Sprache beheimatete Gedächtnis, die in ihr innewohnende kollektive Erfahrung der Slowenen, in die deutsche Sprache zu transferieren. (Haderlap 2013)

Die Zeugen und Zeugnisse der historischen Erfahrung der Kärntner Slowenen verschwinden unweigerlich und die slowenische Sprache in Kärnten droht ebenfalls zu sterben, so dass sich die Frage stellt, welche Form das kulturelle Gedächtnis annehmen kann. Zuvor stellt sich überhaupt die Frage, was Zeugnis für solch ein Grauen sein kann - als das Mädchen nach den Erzählungen der Großmutter deren Körper absucht, „ob vom Entsetzen Spuren auf der Haut geblieben sind“, stellt sie fest, „das Grauen zeichnet sich nicht ab“ (Haderlap 2011: 122). Der Großmutter jedoch gelingt es, indem sie „alle nachbarlichen Anwesen mit den ehemals dort ansässigen Menschen [...], die das Lager überlebt haben oder in den Lagern ums Leben gekommen sind“, aufzählt und auf diese Weise „ein feines Netz von Hof zu Hof“ (Haderlap 2011: 141) flicht. ${ }^{18}$ Dem folgt im Roman ein Absatz, in dem die Namen der Großmutter geradezu beschwörend aufgezählt werden. Es entsteht ein literarischer Gedenkstein slowenischer Namen, der in das Archiv nicht nur der deutschen Sprache eingehen wird.

Zum dritten Punkt: Da Maja Haderlap zweisprachig ist, wirkt, wenn sie in einer Sprache schreibt, die andere Sprache im Hintergrund mit, weil die Sprachen im Sprachzentrum untereinander vernetzt sind. Wer mehrsprachig ist, kann nicht mehr rein einsprachig denken. Haderlap selbst meint hierzu: „In meiner Schreibbewegung gedenke ich immer wieder meiner slowenischen Erstsprache." (Haderlap 2013) Als Beispiel nennt sie das Gedicht gedächtnis, vergissmeinicht, monument.

[...] in meiner stimme

kristallisiert die erste sprache und

memoriert die chiffren der erinnerung:

spomin, spominčica, spomenik.

gedächtnis, vergissmeinnicht, monument. (Haderlap 2014: 75)

18 Das geflochtene Netz kann auch mit dem gesponnenen Trichter in der letzten Passage des Romans verbunden werden. Offen bleibt jedoch hier wie dort, wie erfolgreich die Erinnerungsarbeit ist. Auch Haderlap spricht bei ihrem Roman von einem Versuch. 
NEIDLINGER, PASEWALCK

Die Chiffren der Wortstämme im Slowenischen erzeugen im Deutschen eine Wortzusammenstellung, in der sich die slowenischen Chiffren semantisch kristallisieren. Dabei entsteht eine spannungsvolle Beziehung zwischen den Sprachen. Eine solche Beziehung ergibt sich allerdings nicht nur durch eine Verknüpfung der Sprachen, sondern auch aus der Aufhebung jeglicher Verknüpfungen, wie sie im semiotischen Dreieck für Sprachen expliziert sind. Danach sind in jeder Sprache die Signifikanten über die Codes (Vorrat an Zeichen) mit den Signifikaten in je spezifischer Weise verbunden, und auf der Grundlage dieser Verknüpfung bezieht man sich auf Phänomene in der Welt. Da jedoch diese Verknüpfungen zwischen den Sprachen nicht gleich sind, begreifen mehrsprachige Menschen, dass Verknüpfungen nur bezogen auf die jeweilige Sprache ihre Gültigkeit besitzen und in Wirklichkeit lediglich eine mögliche Welt darstellen. Einerseits entsteht hierdurch eine Unsicherheit, weil die Sprachregeln, die Gebote und Verbote einer Sprache zu bloß möglichen Sprachkonventionen werden, und zudem die Gefahr besteht, dass man die unterschiedlichen Vereinbarungen der Sprachen vermischt oder verwechselt. Andererseits entsteht in diesem Niemandsland zwischen den Sprachen, in dem die Verknüpfungen ihre Selbstverständlichkeit verlieren, im Schreibakt eine Freiheit zur Verknüpfung. Silke Pasewalck hat hierfür - am Beispiel der Autorin Ilma Rakusa - den Begriff „Mehr-Sprachlichkeit ${ }^{“ 19}$ vorgeschlagen. Maja Haderlap bezieht sich auf Ilma Rakusa und erläutert diesen Raum bzw. Ort der Sprache wie folgt:

19 Diesen Terminus hat Silke Pasewalck ergänzend zu Robert Stockhammers ,Sprachigkeit' vorgeschlagen, um hervorzuheben, dass es sprachliche Elemente oder sprachliche Strukturen geben kann, bei denen erstens der Grad der Zugehörigkeit nicht klar ist und die zweitens auf eine Sprache verändernd einwirken können. Ihre Sprachigkeit ist unterbestimmt, weil sie einerseits eine Verfremdung bezogen auf eine Sprache aufweisen, andererseits diese nur in einer Sprache realisieren können. Maja Haderlap formuliert dies in aller Deutlichkeit: „Es ist doch so, dass man nicht zwischen den Sprachen schreibt, sondern nur in der Sprache schreiben [...] kann." (Haderlap 2011b) In einer Sprache gibt es also ein ,Mehr' als die Sprachigkeit, etwas, das nicht mehrsprachig, sondern ,mehrsprachlich' ist, bei dem ein Sprachausdruck eben nicht nur in der Zugehörigkeit graduell zwischen zwei Sprachen zu verorten ist, sondern in einer Sprache neue Formen und Bedeutungspotentiale von Sprache eröffnet. Zweitens zeigt der Terminus mit seiner typographischen Zuspitzung auf, dass mehr-sprachliche Elemente oder Strukturen auf eine Sprache einwirken und diese kreativ verändern bzw. deren Grenzen erweitern können. Dies meint unseres Erachtens auch Haderlap, wenn sie davon spricht, dass sie sich eine Sprache erschreibe oder erfinde. Womit eben keine Konstruktion einer neuen Sprache, sondern - in Anlehnung an den russischen Formalismus - Poetizität gemeint ist. Vgl. hierzu Pasewalck 2014 und Pasewalck 2018. 
„Die Sprache hat also ihren Ort."

Ilma Rakusa hat einmal geschrieben, dass man als Mehrsprachige lernt, dass es nichts Selbstverständliches gibt, dass alles auf Differenz beruhe. Es gibt keinen Ort, der so sehr nach einem Wortwechsel, nach einer Übersetzung verlangt wie die Sprachgrenze. Einen solchen Ort, vielmehr einen solchen Raum, bewohne auch ich. Er ist nicht sichtbar und gleicht einem verdunkelten Korridor, den ich als Verbindungsweg zwischen meine bestimmenden Sprachen gebaut oder gegraben habe. [...] Im Korridor lege ich alles Bezeichnende und Bezeichnete $a b$, werde frei von Zuschreibungen. Außerhalb des Korridors sehe ich die Sprachen leuchten. (Haderlap 2011b)

\section{Silke Pasewalck}

silke.pasewalck@bkge.uni-oldenburg.de

Bundesinstitut für Kultur und Geschichte der Deutschen im östlichen Europa /

Federal Institute for Culture and History of the Germans in Eastern Europe GERMANY

\section{Dieter Neidlinger}

klausn@ut.ee

\section{Literaturverzeichnis}

Burz, U. 2014. Kärnten. - ome-lexikon.uni-oldenburg.de/54127.html (17.07.2020).

Dembeck, T. 2017. Sprachwechsel/Sprachmischung. - T. Dembeck, R. Parr, eds., Literatur und Mehrsprachigkeit. Ein Handbuch. Tübingen: Narr Francke Attempto Verlag, 125-166.

Haderlap, M. 2011. Engel des Vergessens. Göttingen: Wallstein Verlag.

Haderlap, M. 2011b. Im Licht der Sprache. Klagenfurter Rede zur Literatur. http://archiv.bachmannpreis.orf.at/bachmannpreis.eu/presse.bachmannpreis. eu/d/11012-1/haderlap_rede.pdf(17.07.2020)

Haderlap, M. im Gespräch mit M. Kerbler 2012, ORF, 09.10.2012. - https://www. youtube.com/watch?v=GCw5_SXUkHk (11.07.2020).

Haderlap, M. 2013. Übergänge, Kakanien - Neue Heimaten. Präsentiert von Corinna Kirchhoff. 23.05.2013. - https://www.yumpu.com/de/document/read/ 22004863/kakanien-neue-heimaten-maja-haderlap-burgtheater (17.07.2020).

Haderlap, M. 2014. langer transit. Gedichte. Göttingen: Wallstein Verlag.

Haderlap, M. 2016. Selbstvorstellung vor der Deutschen Akademie für Sprache und Dichtung. - https://www.deutscheakademie.de/de/akademie/mitglieder/majahaderlap/selbstvorstellung (17.07.2020).

Martyn, D. 2014. Es gab keine Mehrsprachigkeit bevor es nicht Einsprachigkeit gab. Ansätze zu einer Archäologie der Sprachigkeit (Herder, Luther, Tawada). - T. 
NEIDLINGER, PASEWALCK

Dembeck, G. Mein, eds., Philologie und Mehrsprachigkeit. Heidelberg: Winter Universitätsverlag, 39-51.

Müller, N. 2006. Einführung in die Mehrsprachigkeitsforschung: Deutsch, Französisch, Italienisch. Tübingen: Narr.

Nebrig, A. 2012. Interlingualität. - E. Zemanek, A. Nebrig, eds., Komparatistik. Berlin: Akademie Verlag, 131-144. https://doi.org/10.1524/9783050059921.131

Pasewalck, S. 2014. „Als lebte ich in einem no man's land, mit Verlaß nur auf die Sprache“. Zu Ilma Rakusas Poetik der Mehrsprachigkeit. - T. Dembeck, G. Mein, eds., Philologie und Mehrsprachigkeit. Heidelberg: Winter Universitätsverlag, 381400.

Pasewalck, S. 2018. Mit Sprache über Sprache hinausgehen. Zum Sprachverständnis bei Wisława Szymborska und Ilma Rakusa. - E. Hess-Lüttich, M. Bhoot, V. Surana, eds., Komparative Ästhtetik(en). Frankfurt/Main: Peter Lang, 245-261.

Prutti, B. 2014. „Ist es nicht ein finsterer Wald, in den wir gerieten?" Waldgänge und Waldgänger in Maja Haderlaps Roman Engel des Vergessens - Studia teodisca, 21, 85-127.

Riehl, C. M. 2004. Sprachkontaktforschung: eine Einführung. Tübingen: Narr.

Schmeling, M. 2004. Multilingualität und Interkulturalität im Gegenwartsroman. M. Schmitz-Emans, ed., Literatur und Vielsprachigkeit. Heidelberg, 221-235.

Steiner, G. 1975. After Babel, aspects of language and translation [Nach Babel. Aspekte der Sprache und des Übersetzens. Übersetzt von Monika Plessner unter Mitwirkung von Henriette Beese, Frankfurt am Main: Suhrkamp, 1992]

Schörkhuber, E. 2017. Zugänge zu einem Archiv der Literatur - entlang der Lektüren von Maja Haderlaps Engel des Vergessens, Bogdan Bogdanovic Die grüne Schachtel. Buch der Träume und Elfriede Jelineks Winterreise. Diss.schrift Universität Wien.

Zemanek, E., Willms, W. 2017. Polyglotte Texte - Einleitung. - E. Zemanek, W. Willms, eds., Polyglotte Texte. Formen und Funktionen literarischer Mehrsprachigkeit von der Antike bis zur Gegenwart, Special Issue, Komparatistik Online, 2, 7-17. 